\title{
On triplet tetraoxygen: ab initio study along minimum energy path and global modelling
}

\author{
A.J.C. Varandas *, J.L. Llanio-Trujillo \\ Departamento de Química, Universidade de Coimbra, P-3049 Coimbra Codex, Portugal
}

Received 11 January 2002; in final form 7 March 2002

\begin{abstract}
MRCI calculations are reported for $\mathrm{O}_{4}\left({ }^{3} \mathrm{~A}\right)$ using two distinct CASSCF reference wave functions, and the results compared with previous ab initio calculations. By combining the force field of the best ab initio CASSCF surface at the saddle point with the recommended estimate for the barrier height, a four-body analytic term is obtained which added to the popular double many-body expansion potential energy surface for the title system makes it fit well such properties. (C) 2002 Published by Elsevier Science B.V.
\end{abstract}

\section{Introduction}

Interest on the triplet state of tetraoxygen can be traced to Chapman's $\mathrm{O}_{x}$ cycle for ozone depletion [1], as it is the supermolecule relevant for studying the elementary reactions involved in it [2]. Recently, it became the focus of much interest and debate in connection to the role of vibrationally excited molecular oxygen in the so-called 'ozonedeficit' problem [3-6]. Despite some controversy [4], it has been suggested that vibrationally hot oxygen molecules $\left[\mathrm{O}_{2}(v)\right]$ may account for the significant discrepancies between observed and predicted ozone concentrations through the reaction

$$
\begin{aligned}
& \mathrm{O}_{2}\left(\mathrm{X}^{3} \Sigma_{\mathrm{g}}^{-}, v \geqslant 26\right)+\mathrm{O}_{2}\left(\mathrm{X}^{3} \Sigma_{\mathrm{g}}^{-}, v=0\right) \\
& \quad \rightarrow \mathrm{O}_{3}+\mathrm{O}\left({ }^{3} \mathrm{P}\right)
\end{aligned}
$$

\footnotetext{
${ }^{*}$ Corresponding author. Fax: +351-39-827-703. das).
}

In fact, $\mathrm{O}_{2}(v)$ molecules are known to be formed during the photodissociation of ozone in the triplet channel

$\mathrm{O}_{3}+h v \rightarrow \mathrm{O}\left({ }^{3} \mathrm{P}\right)+\mathrm{O}_{2}\left(\mathrm{X}^{3} \Sigma_{\mathrm{g}}^{-}, v \geqslant 26\right)$,

and measurements [5,7] of the total depletion rate of $\mathrm{O}_{2}(v)$ in collisions with molecular oxygen have shown a sharp increase above $v=25$ suggesting that it could be due to reaction (1). However, such an observation has not been fully explained experimentally $[5,6]$. The caveat extends also to theoretical ([8-13], and references therein) work, most of which employed our own global $\mathrm{O}_{4}$ potential energy surface [14] obtained from the double many-body expansion (DMBE) $[2,15,16]$ method. Although recent spectroscopic studies [17] and theoretical calculations [18] suggest that nonadiabatic processes may also be relevant to explain such a process, there are important issues concerning the ground state potential energy surface that deserve further study. 
In this work, we address two such issues. First, we provide a comparative study of the title system along its minimum energy path (MEP) using ab initio calculations with large multiconfigurational wave functions. Previous electronic structure calculations on $\mathrm{O}_{4}\left({ }^{3} \mathrm{~A}\right)$ have been carried out by Lauvergnat and Clary (LC) [11], and HernándezLamoneda and Ramirez-Solis (HR) [18]. The former used the internally contracted MRCI method based on a $\operatorname{CASSCF}(6,6)$ reference wave function ${ }^{1}$ and a [6-311G] Pople-type basis set [20] with addition of polarization functions for high level of correlation [11] $([6-311 \mathrm{G}](2 \mathrm{~d}, \mathrm{f})$, which is a double$\zeta$ basis on the $1 \mathrm{~s}$ and $2 \mathrm{~s}$ atomic orbitals and triple- $\zeta$ on the $2 \mathrm{p}$ atomic orbitals). Thus, the active space consisted of four molecular orbitals $\left(\sigma_{\mathrm{bc}}, \sigma_{\mathrm{bc}}^{*}, \sigma_{\mathrm{cd}}\right.$, and $\sigma_{\text {cd }}^{*}$, where the small capitals label the oxygen atoms) associated to two selected active bonds $\left(\mathrm{O}_{\mathrm{b}}-\mathrm{O}_{\mathrm{c}}\right.$ and $\left.\mathrm{O}_{\mathrm{c}}-\mathrm{O}_{\mathrm{d}}\right)$, and two orbitals associated with the two unpaired electrons of the triplet state. Then, dynamical correlation was included by considering all single and double excitations out from the CAS $(6,6)$ reference wave function, while the Davidson correction [21] was used to take into account approximately the effects of higher excitations. In their studies, two MRCI calculations have been tested. In $\mathrm{MRCI}_{1}$, the first 13 doubly occupied MOs were not correlated, while in $\mathrm{MRCI}_{2}$ the first four MOs (the four core orbitals of oxygen) were left uncorrelated. As noted by LC [11] themselves, since the $\sigma_{a b}$ bond was always kept doubly occupied, the two oxygen molecules obtained for the $\mathrm{O}_{2}+\mathrm{O}_{2}$ dissociation limit are unfortunately not equivalent, and their optimized bond lengths are different. In other words, when the $\mathrm{O}_{\mathrm{b}}-\mathrm{O}_{\mathrm{c}}\left(R_{2}\right)$ and $\mathrm{O}_{\mathrm{c}}-\mathrm{O}_{\mathrm{d}}\left(R_{3}\right)$ distances are large, the CAS calculation reduces to a restricted openshell Hartree-Fock calculation [22]. Of course, this limitation can be avoided by increasing the active space, although this may become prohibitive if a large number of points for the description of the potential energy surface is required. For example, HR [18] employed a reference wave function in

\footnotetext{
${ }^{1}$ The acronym $\operatorname{CASSCF}(m, n)$ stands for complete active space self-consistent field [19], hereafter denoted $\operatorname{CAS}(m, n)$, where $m$ and $n$ denote the number of active electrons orbitals and active molecular orbitals.
}

which only the $1 \mathrm{~s}$ and $2 \mathrm{~s}$ orbitals were kept doubly occupied in all configurations (this corresponds to take $m=16$ and $n=12$ ), and employed a $5 \mathrm{~s} 4 \mathrm{p} 3 \mathrm{~d} 2 \mathrm{f}$ ANO basis set. Without symmetry, this leads to expansions of 113256 configuration state functions (CSFs). Using such a CAS $(16,12)$ reference wave function, they then included the dynamical correlation through second-order multireference perturbation theory [i.e., CAS $(16,12)+$ MP2]. With a view to perform MRCI calculations on $\mathrm{O}_{4}$ and larger systems containing $\mathrm{O}_{x}(x \geqslant 4)$ as a fragment, it will be valuable to explore the smallest active-space sizes which can allow a proper description of all relevant dissociation channels. This will be done in this work for the lowest triplet state of tetraoxygen. The second issue of concern here is to provide a four-body term that added to the DMBE potential energy surface (heretofore referred to as DMBE I) brings it into close agreement with the ab initio calculations at the transition state for reaction (1).

The Letter is organized as follows. In Section 2, we describe the ab initio methods and compare the results obtained to those previously reported. Section 3 presents the strategy adopted to determine the four-body correction term to be added to the $\mathrm{O}_{4}$ DMBE I surface. The conclusions are gathered in Section 4.

\section{Ab initio calculations}

\subsection{General}

For the title system, a full valence complete active space (FVCAS) calculation comprises as much as 24 electrons distributed among 16 active orbitals, amounting to 1485120 CSFs. Although this is impracticable, it may also not be necessary, especially having in mind that sizeable errors may persist due to incompleteness of the one-electron basis sets ([23], and references therein). In fact, to obtain a global potential energy surface for a polyatomic molecule one should carefully select the method and geometries to be calculated, especially if the system has four atoms or more. (An alternative strategy which avoids calculation of the global potential energy surface is ab initio reaction 
dynamics, although this is a very time consuming approach; see, e.g., [24] and references therein.) For example, in a chemical reaction, the most important effort should normally concentrate in regions associated with formation and breaking of chemical bonds (normally one or two). The calculations can then be performed by choosing as active orbitals only those taking part in the bondbreaking/bond-forming process. Such an idea goes back to the pioneering work of Ruedenberg and Sundberg [25], and fits well within the philosophy of the CAS [19] model. Of course, only semiquantitative results can be expected from such an approach. In fact, the CAS model has not been developed for treating dynamical correlation effects, but to provide a good zero-order approximation to the wavefunction. For better accuracy, it will be essential to include the dynamical correlation either variationally (MRCI) or perturbatively (CAS+MP2). Even at these levels, some semiempirical scaling of the dynamical correlation $[26,27]$ may turn out to be crucial for agreement with experiment. In summary, a balanced effort through a careful choice of the size of the active space may turn out to be essential for bringing the problem to realization. For example, the LC choice of six active valence electrons and six active MOs leads to only $189 \mathrm{CSFs}$, although the price to pay is the unphysical behavior of $\mathrm{O}_{4}\left({ }^{3} \mathrm{~A}\right)$ upon dissociation in two oxygen molecules. The immediate question is therefore what is the smallest size of the active spaces capable of removing such a limitation. As it will be shown below, it may suffice to take $m=n=8$ which leads to 2352 CSFs.

\subsection{Results and discussion}

All calculations except CAS $(16,12)$, which have been carried out using Gamess [28], have been done using the Molpro [29] suite of programs. Two levels of accuracy have been considered when performing the MRCI calculations with the CAS $(8,8)$ referecence wave function. In $\mathrm{MRCI}_{1}$, the first 12 doubly occupied MOs have been kept uncorrelated, while in $\mathrm{MRCI}_{2}$ only the first eight doubly occupied MOs were left uncorrelated. This is a more realistic level, since in the oxygen atom the energy gap between the $2 \mathrm{~s}$ and $2 \mathrm{p}$ levels is quite large $(\sim 16 \mathrm{eV})$ compared with the energies normally encountered in simple chemical reactions. Thus, it seems plausible to assume that only the four $2 \mathrm{p}$ electrons on the $\mathrm{O}$ atoms will be involved in the bond-breaking/bond-forming process. Of course, one can go further and leave uncorrelated only the four core orbitals of the oxygen atoms, or stop at the intermediate size employed by HR [18]. Yet, there are strong requirements of CPU time even in $\mathrm{MRCI}_{2}$, and hence such a correlation level has been abandoned. CAS $(16,12)$ calculations have also been carried out using the [6-311G] $(2 \mathrm{~d}, \mathrm{f})$ basis set to compare with the HR results. For this CAS too, MRCI calculations were computationally unaffordable using the means at our disposal.

To test the $\mathrm{MRCI}_{1}$ and $\mathrm{MRCI}_{2}$ models, we have studied the MEP for the reaction

$$
\mathrm{O}_{3}+\mathrm{O} \rightarrow \mathrm{O}_{4}(\mathrm{TS}) \rightarrow \mathrm{O}_{2}+\mathrm{O}_{2}
$$

which is here identified with the distinguished reaction path (DRP) calculated using Molpro [29]. Thus, rather than following a steepest descent path in mass weighted coordinates starting at the saddle point both in the forward (toward the products) and backwards (toward the reactants) directions (so-called intrinsic reaction path), we have selected an internal coordinate (distinguished coordinate) as an approximate reaction coordinate and, at a number of values of this coordinate covering from the saddle point to a large separation at the reactants and products, performed a constrained geometry optimization with respect to all other internal coordinates. Note that the computational cost of this procedure may not simply be proportional to the number of points originally chosen: the more widely spaced are the values of the distinguished coordinate, the more computation time may be required for the geometry optimization. Calculations have been carried out both at the CAS $(6,6)$ (thus, we have repeated the LC [11] calculations) and $\operatorname{CAS}(8,8)$ levels, based on the following steps. First, we have determined the transition-state (TS) geometry at the CAS $(\mathrm{m}, \mathrm{m}) / 6$ $311 \mathrm{G}(2 \mathrm{~d}, \mathrm{f})$ levels $(m=6,8)$ using the transition state of the DMBE I [14] potential energy surface as starting guess. Such CAS calculations have been found to converge very well, especially if one switches-off the coupling between the CI 
coefficients and orbital rotations during the first few iterations. The calculated structures correspond at both CAS levels to genuine saddle points as seen from the vibrational frequencies shown in Table 1. We emphasize that the DMBE I transition-state structure provided a good starting point, a finding also noted by LC [11]. In fact, only 20 geometry optimization steps were required to reach the saddle point in the ab initio calculations. For each value of the distinguished reaction coordinate $\left[R\left(\mathrm{O}_{\mathrm{b}}-\mathrm{O}_{\mathrm{c}}\right)\right.$ for $\mathrm{O}_{4}(\mathrm{TS}) \rightarrow \mathrm{O}_{2}+\mathrm{O}_{2}$, and $R\left(\mathrm{O}_{\mathrm{c}}-\mathrm{O}_{\mathrm{d}}\right)$ for $\mathrm{O}_{4}(\mathrm{TS}) \rightarrow \mathrm{O}_{3}+\mathrm{O}$ path], the geometries were fully optimized with respect to all other degrees of freedom. The MEPs calculated at the various levels of theory are shown in Fig. 1, while Tables 1 and 2 gather the major numerical results of the present work. Clearly, the two oxygen molecules at the $\mathrm{O}_{2}+\mathrm{O}_{2}$ dissociation channel are not equivalent at the CAS $(6,6)$ level as shown by the different optimized bond lengths [11]. Also notable is the fact that the $C_{2 \mathrm{v}}$ symetry of the equilibrium $\mathrm{O}_{3}$ molecule [which is lost when using a $\operatorname{CAS}(6,6)$ reference wave function] has beenrecovered at the $\operatorname{CAS}(8,8)$ level of theory.

The salient features in both the $\operatorname{CAS}(6,6)$ and CAS $(8,8)$ MEPs are the barrier in the $\mathrm{O}+\mathrm{O}_{3}$ channel $\left(\mathrm{TS}_{1}\right)$, and the appearance of a deep $\left(\mathrm{TS}_{2}\right)$ at $\operatorname{CAS}(8,8)$ level after the barrier toward the $\mathrm{O}_{2}+\mathrm{O}_{2}$ channel. In fact, both such structures correspond to transition states of index one on the potential energy surface, as seen from the existence of only one imaginary vibrational frequency. The fact $\mathrm{TS}_{1}$ and $\mathrm{TS}_{2}$ are directly connected along the MEP would suggest that symmetry is lost somewhere between those two points [30]. Such a belief seems to be supported by Fig. 2 which shows stick

Table 1

Calculated geometries, frequencies, and zero-point energies for the stationary points of the $\mathrm{O}_{4}\left({ }^{3} \mathrm{~A}\right)$ potential energy surface

\begin{tabular}{|c|c|c|c|c|c|c|}
\hline \multirow{2}{*}{$\begin{array}{l}\text { Stationary } \\
\text { point }\end{array}$} & \multicolumn{3}{|l|}{ Geometries $^{\mathrm{a}}$} & \multicolumn{3}{|l|}{ Frequencies $^{b}$} \\
\hline & $\operatorname{CAS}(6,6)^{\mathrm{c}}$ & $\operatorname{CAS}(8,8)$ & $\operatorname{CAS}(16,12)^{\mathrm{d}}$ & $\operatorname{CAS}(6,6)^{\mathrm{c}}$ & $\operatorname{CAS}(8,8)$ & $\operatorname{CAS}(16,12)^{\mathrm{d}}$ \\
\hline $\mathrm{O}_{2}+\mathrm{O}_{2}$ & $\begin{array}{l}R_{1}=2.1764 \\
R_{3}=2.2047\end{array}$ & $\begin{array}{l}R_{1}=2.2048 \\
R_{3}=2.2048\end{array}$ & $\begin{array}{l}R_{1}=2.2928 \\
R_{3}=2.2928\end{array}$ & $\begin{array}{l}2012.6(2004.5) \\
1896.4(1867.1) \\
5.59^{\mathrm{e}}\end{array}$ & $\begin{array}{l}1874.1 \\
1874.3 \\
5.36^{\mathrm{e}}\end{array}$ & $\begin{array}{l}1558.8 \\
1558.8 \\
4.46\end{array}$ \\
\hline $\mathrm{O}_{3}+\mathrm{O}$ & $\begin{array}{l}R_{1}=2.3119 \\
R_{2}=2.4493 \\
\theta_{1}=115.6\end{array}$ & $\begin{array}{l}R_{1}=2.4153 \\
R_{2}=2.4153 \\
\theta_{1}=114.9\end{array}$ & $\begin{array}{l}R_{1}=2.4208 \\
R_{2}=2.4211 \\
\theta_{1}=116.54\end{array}$ & $\begin{array}{l}1419.2(1413.5) \\
1000.6(996.8) \\
759.3(756.4) \\
4.54^{\mathrm{e}}(4.53)\end{array}$ & $\begin{array}{c}1222.9 \\
1002.9 \\
731.0 \\
4.23^{\mathrm{e}}\end{array}$ & $\begin{array}{l}1107.8 \\
1064.4 \\
714.8 \\
4.13\end{array}$ \\
\hline $\mathrm{O}_{4}\left(\mathrm{TS}_{1}\right)$ & $\begin{array}{l}R_{1}=2.3678 \\
R_{2}=2.6052 \\
R_{3}=3.7708 \\
\theta_{1}=111.35 \\
\theta_{2}=104.06 \\
\phi=86.29\end{array}$ & $\begin{array}{l}R_{1}=2.5051 \\
R_{2}=2.5574 \\
R_{3}=3.7517 \\
\theta_{1}=110.13 \\
\theta_{2}=105.02 \\
\phi=86.39\end{array}$ & $\begin{array}{l}2.4088(2.44) \\
2.6503(2.65) \\
3.4923(3.52) \\
112.45(112.8) \\
105.31(106.1) \\
85.13(83.0)\end{array}$ & $\begin{array}{l}583.2 \mathrm{i}(581.0 \mathrm{i}) \\
126.7(126.3) \\
275.9(274.9) \\
611.9(609.6) \\
692.6(690.0) \\
1202.7(1198.2) \\
4.16^{\mathrm{e}}\end{array}$ & $\begin{array}{l}573.7 \mathrm{i} \\
128.6 \\
286.1 \\
644.1 \\
670.7 \\
935.9 \\
3.81^{\mathrm{e}}\end{array}$ & $\begin{array}{l}702.6 \mathrm{i}(756 \mathrm{i}) \\
139.6(139) \\
307.9(306) \\
494.9(491) \\
669.2(668) \\
1105.3(1051) \\
3.88^{\mathrm{e}}(3.80)\end{array}$ \\
\hline $\mathrm{O}_{4}\left(\mathrm{TS}_{2}\right)$ & & $\begin{array}{l}R_{1}=2.5155 \\
R_{2}=2.8141 \\
R_{3}=2.5155 \\
\theta_{1}=106.6 \\
\theta_{2}=106.6 \\
\phi=79.2\end{array}$ & & & $\begin{array}{l}273.0 \mathrm{i} \\
349.6 \\
576.4 \\
648.2 \\
722.1 \\
891.8 \\
4.56^{\mathrm{e}}\end{array}$ & \\
\hline
\end{tabular}

\footnotetext{
${ }^{\mathrm{a}}$ In Bohr and degrees.

${ }^{\mathrm{b}}$ In $\mathrm{cm}^{-1}$.

${ }^{\mathrm{c}}$ Given in parenthesis are the results from [11]; within the accuracy reported, the geometries are identical to those calculated in the present work.

${ }^{\mathrm{d}}$ Given in parenthesis are the results from [18].

${ }^{\mathrm{e}}$ Zero-point energy in $\mathrm{kcal} \mathrm{mol}{ }^{-1}$.
} 


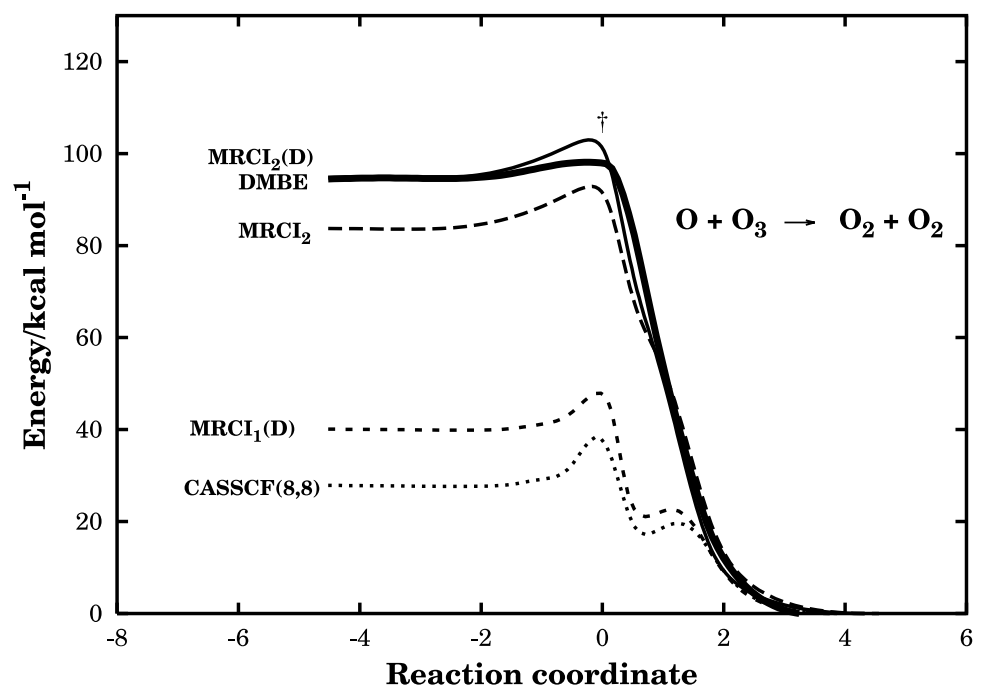

Fig. 1. DRP for the process $\mathrm{O}_{3}+\mathrm{O} \rightarrow \mathrm{O}_{4}(\mathrm{TS}) \rightarrow \mathrm{O}_{2}+\mathrm{O}_{2}$ at various theoretical levels: thin solid line $\mathrm{MRCI}$ (D); long-dash line, $\mathrm{MRCI}_{2}$; short-dash line, $\mathrm{MRCI}_{1}$ [this is indistinguishable from $\mathrm{MRCI}_{1}(\mathrm{D})$ within the scale of the figure]; dotted line, CAS(8,8). Also shown for comparison is the MEP of the $\mathrm{O}_{4}$ DMBE I [14] potential energy surface (solid bold line).

Table 2

Calculated energies in $E_{\mathrm{h}}$, and dissociation energies relative to $\mathrm{O}_{2}+\mathrm{O}_{2}$ in kcal mol ${ }^{-1}$

\begin{tabular}{|c|c|c|c|c|c|c|c|c|}
\hline \multirow[t]{2}{*}{ Species } & \multicolumn{3}{|l|}{ CASSCF } & \multicolumn{3}{|c|}{ MRCI/CAS $(6,6)$} & \multicolumn{2}{|c|}{$\mathrm{MRCI} / \mathrm{CAS}(8,8)$} \\
\hline & $\operatorname{CAS}(6,6)$ & $\operatorname{CAS}(8,8)$ & $\operatorname{CAS}(16,12)$ & $\mathrm{CI}_{1}(13)$ & $\mathrm{CI}_{2}(8)$ & $\mathrm{CI}_{3}(4)$ & $\mathrm{CI}_{1}(12)$ & $\mathrm{CI}_{2}(8)$ \\
\hline $\mathrm{O}_{3}+\mathrm{O}$ & -299.261207 & -299.302963 & -299.361103 & & & -299.953136 & -299.380799 & -299.689672 \\
\hline $\mathrm{O}_{3}+\mathrm{O}(\mathrm{D})^{\mathrm{a}}$ & & & & & & -300.049390 & -299.382204 & -299.728280 \\
\hline $\mathrm{O}_{2}+\mathrm{O}_{2}$ & -299.324263 & -299.347876 & -299.487181 & -299.379609 & -299.7940999 & -300.079203 & -299.444441 & -299.823652 \\
\hline $\mathrm{O}_{2}+\mathrm{O}_{2}(\mathrm{D})^{\mathrm{a}}$ & & & & -299.380322 & -299.8473608 & -300.192459 & -299.446554 & -299.879741 \\
\hline $\mathrm{TS}_{1}$ & -299.246708 & -299.287623 & -299.335983 & -299.289146 & -299.6435825 & -299.936619 & -299.368685 & -299.668578 \\
\hline $\mathrm{TS}_{1}(\mathrm{D})^{\mathrm{a}}$ & & & & -299.289651 & -299.6848469 & -300.034896 & -299.370375 & -299.708472 \\
\hline $\mathrm{TS}_{2}$ & & -299.320312 & & & & & & \\
\hline $\mathrm{TS}_{1}^{\mathrm{b}}$ & $48.66,47.23^{c}$ & $37.7,36.15^{\mathrm{c}}$ & $94.88,94.30^{\mathrm{c}}$ & $56.77,55.34^{\mathrm{c}}$ & $94.45,93.02^{\mathrm{c}}$ & $89.47,88.04^{\mathrm{c}}$ & $47.53,45.98^{\mathrm{c}}$ & $97.31,95.76^{\mathrm{c}}$ \\
\hline $\mathrm{TS}_{1}(\mathrm{D})^{\mathrm{b}, \mathrm{c}}$ & & & & $56.89,55.46^{\mathrm{c}}$ & $101.98,100.55^{\mathrm{c}}$ & $98.87,97.44^{\mathrm{c}}$ & $47.80,46.25^{\mathrm{c}}$ & $\begin{array}{l}107.47 \\
105.92^{\mathrm{c}}\end{array}$ \\
\hline
\end{tabular}

\footnotetext{
${ }^{\mathrm{a}}$ Including the Davidson correction [22].

${ }^{\mathrm{b}}$ Relative to $\mathrm{O}_{2}+\mathrm{O}_{2}$.

${ }^{\mathrm{c}}$ With zero-point energy correction.
}

and ball pictures of the $\operatorname{CAS}(8,8)$ structures associated to $\mathrm{TS}_{1}$ and $\mathrm{TS}_{2}$. While $\mathrm{TS}_{1}$ is found to have $\mathrm{C}_{1}$ symmetry, $\mathrm{TS}_{2}$ is instead found to have symmetry $\mathrm{C}_{2}$. We should emphasize that all calculations have been carried out without symmetry requirements, and hence the symmetry of $\mathrm{TS}_{2}$ is genuine and simply dictated by the energy minimization criterion. We now recall that bifurcations may, in particular, occur when $\mathrm{C}_{2}$ or a higher symmetry is lost [31]. This can easily be recognized by noting that two products are formed when moving backwards from $\mathrm{TS}_{2}$ to $\mathrm{TS}_{1}$ which are transformed by the $\mathrm{C}_{2}$ operation, whereas there is only one reactant species $\left(\mathrm{TS}_{2}\right)$ which goes into itself under such an operation. It seemed therefore likely that the path of reaction would first follow a valley on the potential energy surface up to somewhere beyond $\mathrm{TS}_{1}$ and then a point occurs 


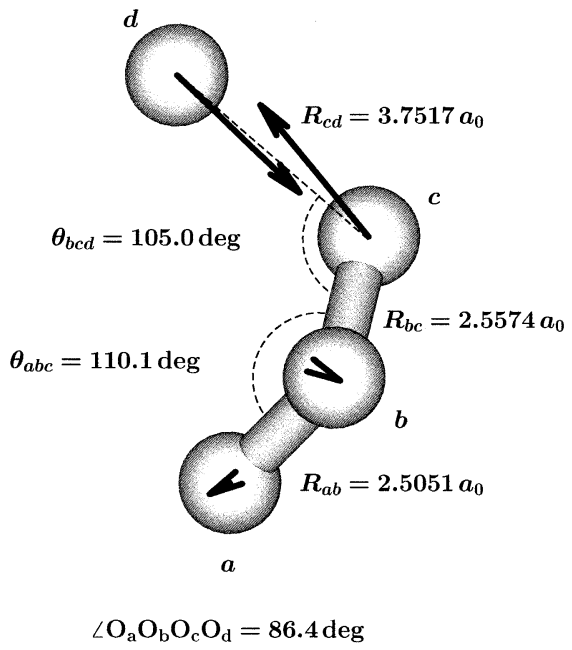

(A)

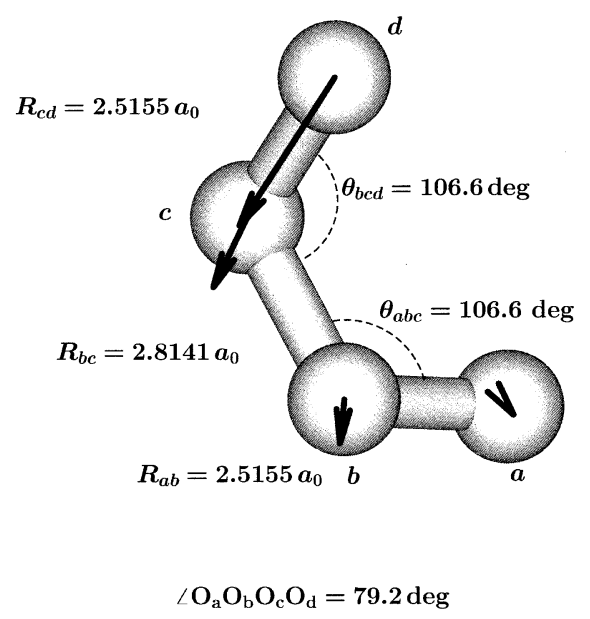

(B)

Fig. 2. Ball and stick structures associated to transition states: (A) $\mathrm{TS}_{1}, \mathrm{CAS}(8,8)$; (B) $\mathrm{TS}_{2}, \mathrm{CAS}(8,8)$. The arrows show the vector displacements associated to the corresponding imaginary frequencies.

where the valley turns into a ridge. The reaction path would then be expected to bifurcate near that point, which is called a valley-ridge inflection point [30] (this VRI point is characterized by having associated a Hessian matrix with a zero eigenvalue, being the corresponding eigenvector perpendicular to the gradient at that point [30]). Such a conjecture seems to find support on the vibrational modes associated to the imaginary frequencies at $\mathrm{TS}_{1}$ and $\mathrm{TS}_{2}$. These are also shown in Fig. 2 by indicating the corresponding vector displacements centered on the atoms. Clearly, in the case of $\mathrm{TS}_{1}$, such vectors indicate that for both the DMBE I (not shown) and CAS $(8,8)$ potential energy surfaces the reactive coordinate corresponds essentially to a vibration of the $\mathrm{O}_{c} \mathrm{O}_{\mathrm{d}}$ bond with $\mathrm{O}_{a} \mathrm{O}_{b}$ acting essentially as a spectactor. In other words, the $\mathrm{TS}_{1}$ structure would reflect the process $\mathrm{O}_{4} \leftrightarrow \mathrm{O}_{3}+\mathrm{O}$ as it occurs early in the reagents valley, while the reactive coordinate in $\mathrm{TS}_{2}$ suggests the process $\mathrm{O}_{4} \leftrightarrow \mathrm{O}_{2}+\mathrm{O}_{2}$ as one diatomic fragment tends to separate from the remaining diatomic. Such an observation suggests that there is a change of reaction coordinate in going from $\mathrm{TS}_{1}$ to $\mathrm{TS}_{2}$. In other words, the DRP shown in Fig. 1 might be a kind of 'top of a ridge' for a certain segment between the VRI point and $\mathrm{TS}_{2}$. Thus, the DRP for such a segment may not be the true MEP, as we may imagine a manifold of paths emanating from this 'top of a ridge' path to the MEP which departs from $\mathrm{TS}_{2}$. Unfortunately, we have been unable to localize such a VRI point, which suggests that $\mathrm{TS}_{2}$ may actually be an artifact due to having used the DRP approach. Although one might conjecture that, if existing, such a structure could affect the inverse reaction (1), we further observe that $\mathrm{TS}_{2}$ (also present when using other basis sets) tends to disappear in the DRP approach when one includes the dynamical correlation. We further observe that no trace of $\mathrm{TS}_{2}$ is found both in the MEP of the DMBE I potential energy surface and the intrinsic reaction coordinate path at the CAS level, including $\operatorname{CAS}(16,12)$.

In an attempt to characterize further $\mathrm{TS}_{2}$, we have performed $\operatorname{CAS}(8,8)$ calculations for the 'cis' and 'trans' structures obtained from $\mathrm{TS}_{2}$ by varying the torsional angle while keeping fixed the other bond lengths and bond angles at their transition state values. The results have shown that both such structures lie above the energy of $\mathrm{TS}_{2}$. Moreover, they do not correspond to stationary points in the $\mathrm{O}_{4}\left({ }^{3} \mathrm{~A}\right)$ potential energy surface, as it has been verified from the analysis of the corresponding gradients. We further observe that the 'trans' conformer is found to have an energy lower than the 'cis' one as might have been anticipated 
from the location of the two lone pairs in the terminal oxygen atoms $[2,11]$.

A final remark goes to the calculated frequencies and classical exothermicities. The notable feature with respect to the former is the very good agreement of the CAS $(16,12)$ results (not reported in [18]) with the experimental values $\left(\mathrm{O}_{2}: 1580 \mathrm{~cm}^{-1}\right.$; $\mathrm{O}_{3}: 1110,1042$, and $\left.705 \mathrm{~cm}^{-1}\right)$. Regarding the classical exothermicities, we obtain from the absolute energies in Table 2 at $\mathrm{MRCI}_{2}$ level without (with) zero-point energy correction the value 84.07 $\mathrm{kcal} \mathrm{mol}^{-1}\left(82.94 \mathrm{kcal} \mathrm{mol}^{-1}\right)$, which are also in fair agreement with the experimental result of 93.64 $\mathrm{kcal} \mathrm{mol}{ }^{-1}$. As seen, the agreement improves considerably when including the Davidson correction: $95.04 \mathrm{kcal} \mathrm{mol}^{-1}\left(94.71 \mathrm{kcal} \mathrm{mol}^{-1}\right)$.

\section{Additional term to correct DMBE surface}

We describe now the construction of a fourbody energy term which, added to DMBE I, brings this into close agreement with the calculated saddle point attributes (geometry and harmonic frequencies) at the $\operatorname{CAS}(16,12)$ level and the best empirical estimate for the barrier height.

First, we describe a procedure for calculating the gradient and Hessian in internal valence coordinates. Consider the relationship between Cartesian displacement coordinates $\xi_{i}$ and internal valence displacement coordinates $S_{\mathrm{t}}$ truncated at linear terms

$S_{\mathrm{t}}=\sum_{i=1}^{3 N} B_{t i} \xi_{i}+\cdots$,

where $B_{t i}$ is an element of the Wilson $\mathbf{B}(M \times 3 N)$ matrix [32]

$B_{t i}=\frac{\partial S_{t}}{\partial \xi_{i}} \quad t=1, \ldots, M, \quad i=1, \ldots, 3 N$,

where $M=3 N-6$. To transform the gradient and Hessian, the standard procedure of Fogarasi and Pulay [33] may then be used. It consists of expanding the energy about a reference configuration using two series, one based upon cartesians and the other internal coordinates. After some algebra, one obtains the following expressions for the internal gradient $\mathbf{g}(M \times 1)$ and force-constant matrix $\mathbf{f}(M \times M)$ in terms of their cartesian counterparts $\mathbf{G}(3 N \times 1)$ and force-constant matrix $\mathbf{F}(3 N \times 3 N)$ :

$\mathbf{g}=\mathbf{A}^{\mathrm{T}} \mathbf{G}$

$\mathbf{f}=\mathbf{A}^{\mathrm{T}} \mathbf{F} \mathbf{A}$

where, $\mathbf{A}(3 N \times M)$ is the generalized inverse of the $\mathbf{B}$ matrix $(\mathbf{B A}=\mathbf{I})$ given by

$\mathbf{A}=\mathbf{u B}^{\mathrm{T}}\left(\mathbf{B u B}^{T}\right)^{-1}$

and $\mathbf{u}(3 N \times 3 N)$ is the well known diagonal matrix formed by the reciprocal of the nuclear masses, each appearing three times on the diagonal. Thus, given a set of cartesian coordinates, the transformations of the gradient and force-constant matrix to internal coordinates requires only the elements of the $\mathbf{B}$ matrix and the masses. To carry out the normal mode analysis, we have employed the standard Wilson $\mathscr{G} \mathscr{F}$ matrix method as outlined by Nguyen et al. [34] (and references therein) with $\mathscr{G}=\mathbf{B u B}^{\mathrm{T}}$ and $\mathscr{F}=\mathbf{f}$.

The correction term to DMBE I potential energy surface is then written as

$$
\begin{aligned}
V_{\text {add }}= & \left(A+c_{1} Q_{1}+c_{2} Q_{1}^{2}+c_{3} Q_{2}+c_{4} Q_{3}\right. \\
& +c_{5} Q_{1}^{3}+c_{6} Q_{1} Q_{2}+c_{7} Q_{1} Q_{3}+c_{8} Q_{4} \\
& +c_{9} Q_{5}+c_{10} Q_{6}+c_{11} Q_{1}^{4}+c_{12} Q_{2}^{2} \\
& +c_{13} Q_{3}^{2}+c_{14} Q_{1}^{2} Q_{2}^{2}+c_{15} Q_{1}^{2} Q_{3}^{2} \\
& +c_{16} Q_{2}^{2} Q_{3}^{2}+c_{17} Q_{1} Q_{4}+c_{18} Q_{1} Q_{5} \\
& +c_{19} Q_{1} Q_{6}+c_{20} Q_{1}^{5}+c_{21} Q_{2} Q_{4} \\
& +c_{22} Q_{2} Q_{5}+c_{23} Q_{2} Q_{6}+c_{24} Q_{3} Q_{4} \\
& \left.+c_{25} Q_{3} Q_{5}+c_{26} Q_{3} Q_{6}+c_{27} Q_{1}^{2} Q_{6}\right) \\
& \times \exp \left[-\gamma \sum_{i=1}^{6}\left(R_{i}-R^{0}\right)^{2}\right],
\end{aligned}
$$

where $R^{0}$ denotes a reference geometry obtained as $R^{0}=\sum_{i=1}^{6} R_{i}^{\mathrm{TS}} / 6$, where $R_{i}^{\mathrm{TS}}(i=1-6)$ denotes the coordinate values at the transition state. In turn, $Q_{i}(i=1-6)$ are totally symmetric combinations of displacement coordinates defined by Varandas and Murrell [35]

$Q_{1}=S_{1}$,

$Q_{2}=S_{2}^{2}+S_{3}^{2}+S_{4}^{2}$, 
Table 3

Numerical values of coefficients ${ }^{\mathrm{a}} A, c_{1} \ldots c_{27}$, and $\gamma$ in Eq. (9)

\begin{tabular}{lll}
\hline$c_{1}=-2.8016957(-1)$ & $c_{10}=-2.6275988$ & $c_{19}=-7.0718575(-3)$ \\
$c_{2}=-1.3130809(-2)$ & $c_{11}=1.0994885(-4)$ & $c_{20}=6.8506571(-6)$ \\
$c_{3}=2.8033750(-1)$ & $c_{12}=-1.0460493(-1$ & $c_{21}=1.8285471(-1)$ \\
$c_{4}=4.3853042$ & $c_{13}=-4.5154863(-1)$ & $c_{22}=-1.6307411(-2)$ \\
$c_{5}=-2.6286855(-5)$ & $c_{14}=4.8807915(-3)$ & $c_{23}=4.1755027(-2)$ \\
$c_{6}=9.7143113(-2)$ & $c_{15}=-2.1796067(-2)$ & $c_{24}=3.0190123(-1)$ \\
$c_{7}=1.6708129(-1)$ & $c_{16}=-2.4883819(-1)$ & $c_{25}=-9.2254026(-2)$ \\
$c_{8}=8.0145511$ & $c_{17}=-1.0954717$ & $c_{26}=8.0964141(-2)$ \\
$c_{9}=1.5600970$ & $c_{18}=-1.0325101$ & $c_{27}=-3.3320016(-3)$ \\
$A=-5.3176637$ & $\gamma=6.5(-1)$ &
\end{tabular}

${ }^{\text {a }}$ For units, see the text.

$Q_{3}=S_{5}^{2}+S_{6}^{2}$,

$Q_{4}=S_{2} S_{3} S_{4}$,

$Q_{5}=S_{6}^{3}-3 S_{6} S_{5}^{2}$,

$Q_{6}=S_{6}\left(2 S_{2}^{2}-S_{3}^{2}-S_{4}^{2}\right)+\sqrt{3} S_{5}\left(S_{3}^{2}-S_{4}^{2}\right)$,

where $S_{i}(i=1-6)$ are the symmetry coordinates:

$S_{1}=\frac{1}{\sqrt{6}}\left(R_{1}+R_{2}+R_{3}+R_{4}+R_{5}+R_{6}\right)$,

$S_{2}=\frac{1}{\sqrt{2}}\left(R_{1}-R_{4}\right)$,

$S_{3}=\frac{1}{\sqrt{2}}\left(R_{2}-R_{5}\right)$,

$S_{4}=\frac{1}{\sqrt{2}}\left(R_{3}-R_{6}\right)$,

$S_{5}=\frac{1}{2}\left(R_{2}-R_{3}+R_{5}-R_{6}\right)$,

$S_{6}=\frac{1}{\sqrt{12}}\left(2 R_{1}-R_{2}-R_{3}+2 R_{4}-R_{5}-R_{6}\right)$.

Note that the fitted data consists of 28 items: 1 energy, six components of the gradient (g), and 21 quadratic force constants (f). The numerical values of the coefficients in Eq. (9) were determined numerically from a least-squares fitting procedure leading to the coefficients reported in Table 3. Table 4 shows that the geometry and barrier height are exactly reproduced while the vibrational frequencies of the corrected DMBE potential en-
Table 4

Geometry $^{\mathrm{a}}$, energy, and vibrational frequencies of the DMBE potential energy surfaces at the saddle point for reaction

\begin{tabular}{lcc}
\hline Property & DMBE I & DMBE II \\
\hline$R_{\mathrm{O}_{\mathrm{a}} \mathrm{O}_{\mathrm{b}}}$ & 2.416 & 2.4088 \\
$R_{\mathrm{O}_{\mathrm{b}} \mathrm{O}_{\mathrm{c}}}$ & 2.514 & 2.6503 \\
$R_{\mathrm{O}_{\mathrm{c}} \mathrm{O}_{\mathrm{d}}}$ & 4.214 & 3.4923 \\
$\theta_{\mathrm{O}_{\mathrm{a}} \mathrm{O}_{\mathrm{b}} \mathrm{O}_{\mathrm{c}}}$ & 117.6 & 112.45 \\
$\theta_{\mathrm{O}_{\mathrm{b}} \mathrm{O}_{\mathrm{c}} \mathrm{O}_{\mathrm{d}}}$ & 139.6 & 105.31 \\
$\phi_{\mathrm{O}_{\mathrm{a}} \mathrm{O}_{\mathrm{b}} \mathrm{O}_{\mathrm{c}}}$ & 118.2 & 85.13 \\
$\mathrm{Energy}^{\mathrm{c}}$ & 97.9 & 97.9 \\
$\omega_{5} / \mathrm{cm}^{-1}$ & 1078.6 & 1176.7 \\
$\omega_{4} / \mathrm{cm}^{-1}$ & 928.2 & 636.8 \\
$\omega_{3} / \mathrm{cm}^{-1}$ & 522.0 & 506.2 \\
$\omega_{2} / \mathrm{cm}^{-1}$ & 322.6 & 225.3 \\
$\omega_{1} / \mathrm{cm}^{-1}$ & 139.4 & 125.0 \\
$\omega_{i} / \mathrm{cm}^{-1}$ & $228.2 \mathrm{i}$ & $712.1 \mathrm{i}$ \\
\hline
\end{tabular}

${ }^{\mathrm{a}}$ The oxygen atoms are labelled by small letters, e.g, $R_{\mathrm{ab}}$ is the bond distance for $\mathrm{O}_{\mathrm{a}} \mathrm{O}_{\mathrm{b}}$. In turn, $\theta_{\mathrm{O}_{\mathrm{a}} \mathrm{O}_{\mathrm{b}} \mathrm{O}_{\mathrm{c}}}$ and $\theta_{\mathrm{O}_{b} \mathrm{O}_{\mathrm{c}} \mathrm{O}_{d}}$ are the angles between $\mathrm{O}_{\mathrm{a}} \mathrm{O}_{b}$ and $\mathrm{O}_{b} \mathrm{O}_{c}$, and $\mathrm{O}_{b} \mathrm{O}_{c}$ and $\mathrm{O}_{c} \mathrm{O}_{d}$, respectively, while $\phi_{\mathrm{O}_{\mathrm{a}} \mathrm{O}_{\mathrm{b}} \mathrm{O}_{\mathrm{c}} \mathrm{O}_{\mathrm{d}}}$ is the dihedral angle.

${ }^{\mathrm{b}}$ This work.

${ }^{\mathrm{c}}$ Relative to $\mathrm{O}_{2}+\mathrm{O}_{2}$.

ergy surface (DMBE II) are within $10 \%$ or so of the calculated CAS $(16,12)$ values, except for $\omega_{1}$ and $\omega_{2}$ which have larger deviations. Note that there is a significant variation in the magnitude of these frequencies for the other CAS calculations (see Table 2), with a tendency to slightly increase with the size of the CAS. Note especially that, like to DMBE I, the DMBE II potential energy surface reproduces the correct exothermicity for the reaction (3).

Fig. 3 shows an energy contour plot of the DMBE II potential energy surface for an oxygen atom orbiting around a non-optimized ozone 


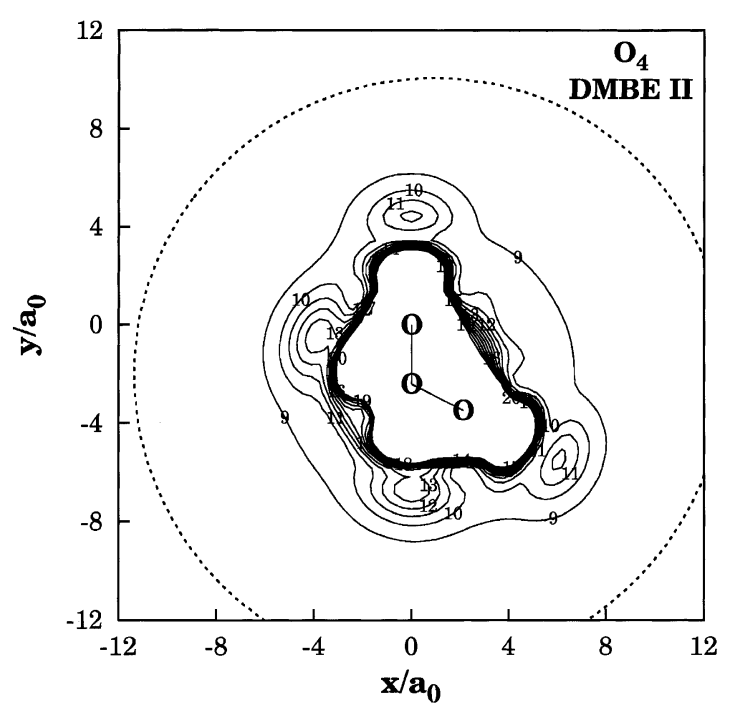

Fig. 3. Energy contour plot of DMBE II potential energy surface for an oxygen atom orbiting around a non-optimized ozone molecule corresponding to plot (b) in [14, Fig. 3]. Contours are equally spaced by $0.02 E_{\mathrm{h}}$ starting at $-0.15 E_{\mathrm{h}}$. The dashed line indicates the energy contour corresponding to the $\mathrm{O}+\mathrm{O}_{3}$ dissociation limit.

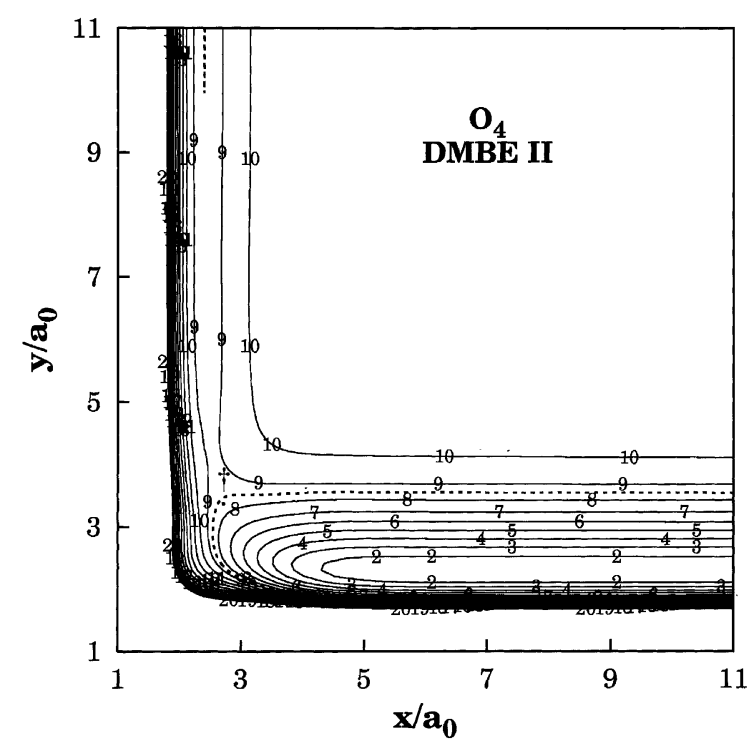

Fig. 4. Energy contour plot of DMBE II potential energy surface for the out-of-plane attack of $\mathrm{O}$ to $\mathrm{O}_{3}$ corresponding to plot (a) in [14, Fig. 5]; $y$ is the distance between the attacking oxygen atom and the terminal oxygen atom of an equilibrium $\mathrm{O}_{3}$ molecule, while $x$ is the distance between the attacked oxygen atom of ozone and the middle one. The $\dagger$ indicates the geometry of the saddle point. molecule. Besides displaying the expected symmetry, a comparison with plot (b) in [14, Fig. 3] shows no visible differences between the two plots. In turn, Fig. 4 shows a contour plot of DMBE II for the out-of-plane attack of $\mathrm{O}$ to $\mathrm{O}_{3}$ corresponding to plot (a) in [14, Fig. 5]. Here too, the differences between DMBE II and the original DMBE I surface can hardly be discerned (the same being true for comparisons referring to the other plots shown in [14]), although the new surface has saddle point properties in closer agreement both with theory and experiment.

\section{Conclusions}

We have carried calculations for $\mathrm{O}_{4}\left({ }^{3} \mathrm{~A}\right)$ using $\operatorname{CAS}(6,6), \operatorname{CAS}(8,8)$, and $\operatorname{CAS}(16,12)$ reference wave functions, with $\operatorname{CAS}(8,8)$ being shown to offer the minimal CAS space for describing all relevant channels of reaction (3). MRCI energies have also been calculated along the reaction path using the first two reference wave functions. Finally, we have recalibrated the four-body term of the previouly reported semiempirical six-dimensional DMBE I [14] potential energy surface to fit the calculated ab initio force-field data, and the best empirical estimate for the barrier height. It would be interesting to see to what extent this affects the dynamics of both the $\mathrm{O}+\mathrm{O}_{3}$ reaction and its reverse in Eq. (1). Work along these lines is in progress in our group.

\section{Acknowledgements}

J.L.L1.T. thanks the Ministerio de Ciencia Tecnologia y Medio Ambiente (Pinar del Rio, Cuba) for leave of absence. The financial support from Fundaçao para a Ciência e Tecnologia, Portugal, under programme PRAXIS XXI is gratefully acknowledged.

\section{References}

[1] S. Chapman, Q. J. R. Metereol. Soc. 3 (1930) 103.

[2] A.J.C. Varandas, Int. Rev. Phys. Chem. 19 (2000) 199. 
[3] R.L. Miller, A.G. Suits, P.L. Houston, R. Toumi, J.A. Mack, A.M. Wodtke, Science 265 (1994) 1831.

[4] P.J. Crutzen, J.U. Grooß, C. Brühl, R. Müller, J.M. Russell III, Science 268 (1995) 705.

[5] C.A. Rogaski, J.A. Mack, A.M. Wodtke, Faraday Discuss. Atmos. Chem. 100 (1995) 229.

[6] D. Stranges, X.M. Yang, J.D. Chesko, A.G. Suits, J. Chem. Phys. 102 (1995) 6067.

[7] J.M. Price, J.A. Mack, C.A. Rogaski, A.M. Wodtke, Chem. Phys. 175 (1993) 83.

[8] R. Hernández-Lammoneda, R. Toumi, D.C. Clary, J. Chem. Phys. 102 (1995) 9544.

[9] N. Balakrishnan, G.D. Billing, Chem. Phys. Lett. 242 (1995) 68.

[10] A.J.C. Varandas, W. Wang, Chem. Phys. 215 (1997) 167.

[11] D. Lauvergnat, D.C. Clary, J. Chem. Phys. 108 (1998) 3566.

[12] N. Balakrishnan, A. Dalgarno, G.D. Billing, Chem. Phys. Lett. 288 (1998) 657.

[13] J. Campos-Martinez, E. Carmona-Novillo, J. Echave, M.I. Hernández, J. Palma, Mol. Phys. 98 (2000) 1729.

[14] A.J.C. Varandas, A.A.C.C. Pais, in: S. Formosinho, I. Czismadia, L. Arnaut (Eds.), Theoretical and Computational Models for Organic Chemistry, Kluwer, Dordrecht, 1991, p. 55.

[15] A.J.C. Varandas, Adv. Chem. Phys. 74 (1988) 255.

[16] A.J.C. Varandas, in: A. Laganá, A. Riganelli (Eds.), Reaction and Molecular Dynamics, Lecture Notes in Chemistry, vol. 75, Springer, Berlin, 2000, p. 33.

[17] R.T. Jongma, S. Shi, A.M. Wodtke, J. Chem. Phys. 111 (1999) 2588.

[18] R. Hernández-Lamoneda, A. Ramírez-Solis, J. Chem. Phys. 113 (2000) 4139.

[19] B.O. Roos, P.E.M. Siegbahn, Plenum Press, New York, 1977, p. 277.
[20] R. Krishnan, J.S. Binkley, J.A. Pople, J. Chem. Phys. 72 (1980) 650.

[21] S.R. Langhoff, E.R. Davidson, Int. J. Quantum Chem. 8 (1974) 61.

[22] E.R. Davidson, Chem. Phys. Lett. 21 (1973) 565.

[23] A.J.C. Varandas, J. Chem. Phys. 113 (2000) 8880.

[24] A.J.C. Varandas, P.E. Abreu, Chem. Phys. Lett. 293 (1998) 261.

[25] K. Ruedenberg, K.R. Sundberg, in: J.-L. Calais, O. Goscinski, J. Linderberg (Eds.), Quantum Science, Plenum Press, New York, 1975, p. 505.

[26] F.B. Brown, D.G. Truhlar, Chem. Phys. Lett. 117 (1985) 307.

[27] A.J.C. Varandas, J. Chem. Phys. 90 (1989) 4379.

[28] M.W. Schmidt, K.K. Baldridge, J.A. Boats, S.T. Elbert, M.S. Gorgon, J.H. Jensen, S. Koseki, N. Matsunaga, K.A. Nguyen, S. Su, T.L. Windus, M. Dupuis, J. Montgomery Jr., J. Comput. Chem. 14 (1993) 1347.

[29] H.-J. Werner, P.J. Knowles, MOLPRO is a package of ab initio programs written by H.-J. Werner, P.J. Knowles, with contributions from J. Almlöf et al., 1998.

[30] P. Valtazanos, K. Ruedenberg, Theor. Chim. Acta 69 (1995) 281.

[31] B.K. Carpenter, J. Am. Chem. Soc. 107 (1985) 5730.

[32] J.E.B. Wilson, J.C. Decius, P.C. Cross, Molecular Vibrations: The Theory of Infrared and Raman Spectra, McGraw-Hill, New York, 1955.

[33] G. Fogarasi, P. Pulay, in: Vibrational Spectra and Structure, 14th edn., Elsevier, Amsterdam, 1985, p. 125.

[34] K.A. Nguyen, C.F. Jackels, D.G. Truhlar, J. Chem. Phys. 104 (1996) 6491.

[35] A.J.C. Varandas, J.N. Murrell, Chem. Phys. Lett. 84 (1981) 440. 